\title{
Studying stellar explosions with Athena
}

\author{
P. O'Brien ${ }^{1}$ and P. Jonker ${ }^{2}$ \\ ${ }^{1}$ Department of Physics and Astronomy, University of Leicester, Leicester, LE1 7RH, UK \\ email: pto2@leicester.ac.uk \\ ${ }^{2}$ SRON, Netherlands Institute for Space Research, Sorbonnelaan 2, 3584-CA, Utrecht, \\ The Netherlands
}

\begin{abstract}
Athena is the second large mission selected in the ESA Cosmic Vision plan. With its large collecting area, high spectral-energy resolution (X-IFU instrument) and impressive grasp (WFI instrument), Athena will truly revolutionise X-ray astronomy. The most prodigious sources of high-energy photons are often transitory in nature. Athena will provide the sensitivity and spectral resolution coupled with rapid response to enable the study of the dynamic sky. Potential sources include: distant Gamma-Ray Bursts to probe the reionisation epoch and find missing baryons in the cosmic web; tidal disruption events to reveal dormant supermassive and intermediate-mass black holes; and supernova explosions to understand progenitors and their environments. We illustrate Athenas capabilities and show how it will be able to constrain the nature of explosive transients including gas metallicity and dynamics.
\end{abstract}

